\title{
Observed Area Under the First Moment Curve Infinity
}

National Cancer Institute

\section{Source}

National Cancer Institute. Observed Area Under the First Moment Curve Infinity. NCI

Thesaurus. Code C85765.

The area under the first moment curve (AUMC) extrapolated to infinity, based on the last observed concentration. 